\title{
Theoretical study on crystal-facet dependency of hydrogen storage rate for shape controlled Pd nano particles
}

\author{
Aya Matsuda ${ }^{1}$ and Hirotoshi Mori ${ }^{2 *}$ \\ ${ }^{1}$ Department of Chemistry and Biochemistry, Graduate School of Humanities and Sciences, \\ Ochanomizu University, 2-1-1 Otsuka, Bunkyo-ku, Tokyo 112-8610, Japan \\ ${ }^{2}$ Faculty of Core Research Natural Science Division, Ochanomizu University, 2-1-1 Otsuka, \\ Bunkyo-ku, Tokyo 112-8610, Japan \\ *Corresponding author. Tel: +81 35978 5718; Fax +8135978 5717 \\ E-mail: mori.hirotoshi@ocha.ac.jp
}

RECEIVED: November 19th, 2015

REVISED: $\quad$ December 7th, 2015 


\begin{abstract}
Pd nano-octahedron absorbs hydrogen gas faster than Pd nano-cube. This means that H-absorption rate of Pd nanoparticles can be controlled by their structural design. For further design of nano materials for $\mathrm{H}$-absorption, it is important to understand tuning mechanism of H-absorption achieved in shape-controlled Pd nano particles. In this study, kinetics of H-absorption into $\mathrm{Pd}$ nano-octahedron and $\mathrm{Pd}$ nano-cube were investigated by electronic structure calculation and molecular dynamics simulation. Both simulations well support experimental observation. It was clarified that the shape dependency in $\mathrm{H}$-absorption of the Pd nanoparticles originates from difference in $\mathrm{H}$-invasion process from surface to the inside.
\end{abstract}




\section{Introduction}

Hydrogen energy is important as novel clean renewable energy. Many scientists and engineers have tried to develop hydrogen storage materials. One of the best known hydrogen absorbing material is bulk Pd. It has been known that bulk Pd can absorb hydrogen gas with the amount of $0.60 \mathrm{H} / \mathrm{Pd}$ at the maximum. Although bulk Pd has good hydrogen absorbing ability within bulk materials, however, it has been considered that the amount of hydrogen absorption is not enough high toward further development for practical use. Even Pd-based materials can absorbs hydrogen at ambient temperature and pressure, further basic research has been strongly required.

With the above background, Yamuchi et al. have reported a fundamental study. To increase the amount of hydrogen storage, it would be important to enlarge effective area for hydrogen absorption on the hydrogen storage materials as much as possible. Based on this consideration, they have tried to synthesize Pd nano particles using chemical reduction of $\mathrm{H}_{2} \mathrm{PdCl}_{4}$ dispersed in solution with PVP (poly[ $N$-vinyl-2-pyrrolidone]) as an protective colloid. They succeeded in preparation of a set of Pd nano particles with diameters of 2.6 \pm 0.4 and $7.0 \pm 0.9 \mathrm{~nm}$. However, contrary to their expectation, they found that the $\mathrm{Pd}$ nano particles does not show better hydrogen absorption properties than the bulk $(0.40-0.50$ $\mathrm{H} / \mathrm{Pd}$ ). Their results indicated that the abilties of hydrogen absorption materials are governed not only by effective surface area but also electronic states of them [1-3] .

As the surface electronic states depend on crystal facet of the materials, it can be considered that we can control the abilities of hydrogen absorption materials by designing their shape. Such a synthesis of shape-controlled nano Pd particles was achieved by Lim et 
al. [4]. They succeeded in the shape control by choosing precursor Pd salts, reducing agent, and capping agent in a careful manner. Applying their techniques, recently, Li et al., synthesized the Pd nano-octahedron and Pd nano-cube, which have only $\{111\}$ or $\{100\}$ facets, and compared their hydrogen absorption properties by means of pressure-composition (PC) isotherms and ${ }^{2} \mathrm{H}-\mathrm{NMR}[5]$. They reported that the rate for hydrogen absorption reaction for the $\mathrm{Pd}$ nano-octahedron is 1.5 times faster than that for $\mathrm{Pd}$ nano-cube at low hydrogen concentration $(\leqq 0.15 \mathrm{H} / \mathrm{Pd})$, though the final amounts of hydrogen absorption at the thermal equilibrium condition are almost same for both particles $(0.48$ and $0.50 \mathrm{H} / \mathrm{Pd}$ at $101.3 \mathrm{kPa}$ for $\mathrm{Pd}$ nano-octahedron and $\mathrm{Pd}$ nano-cube, respectively). However, the rate tuning mechanism for the hydrogen absorption reaction has not been clear, since there has been no theoretical report on the experimental results.

The object of this study is to reveal the origin of the difference in the hydrogen absorption rates between $\mathrm{Pd}$ nano-octahedrons and $\mathrm{Pd}$ nano-cubes. Using density functional theory (DFT) calculations and molecular dynamics (MD) simulations, hydrogen absorption kinetics of shape controlled Pd nanoparticles were theoretically investigated.

\section{Computational Details}

The facet dependency of the hydrogen absorption rate of Pd nano particles was investigated theoretically. In this letter, we discuss the difference in hydrogen absorption kinetics between Pd nano-octahedrons with $\{111\}$ facet and Pd nano-cubes with $\{100\}$ facet. It has been known that hydrogen absorption of bulk Pd consists of the next three steps; (1) dissociative absorption of hydrogen molecules on Pd surface, (2) invasion of hydrogen atoms 
from the Pd surface to octahedral sites located in subsurface, and (3) diffusion of the hydrogen atoms into octahedral sites located in further inside of the Pd lattice. Here, we mainly focus on the processes (2) and (3), since it has been experimentally confirmed that the process (1) is not rate-determining step above $200 \mathrm{~K}[6]$. As the experimental results focused here were observed at 303K [5], it is more important to understand processes (2) and (3) than the process (1). In order to investigate processes (2) and (3) in detail, we performed two types of theoretical simulations explained in the following paragraphs.

The first approach we applied is quantum chemical calculation. For this approach Turbomole 6.4 program package [7] was used. To investigate the differences in hydrogen absorbing potentials for Pd nano-octahedrons with $\{111\}$ facet and Pd nano-cubes with $\{100\}$ facet, we performed a set of density functional theory (DFT) calculations employing PBE density functional $[8,9]$. Using valence only def-SV(P) basis sets with relativistic pseudo potentials, scalar relativistic effects for valence electrons were taken into account. To reduce computational costs, the resolution of identity (RI) approximation [10] for two-electron integrals was also applied to the DFT calculations. It should be also noted that the electronic structures of the systems we focus here are metallic. In this study, we took into account thermal excitations of the valence electrons using pseudo-Fermi smearing technique [11]. To evaluate electronic states of different facets in an equal level, we used a model cluster shown in Figure 1. The model cluster consists of cubooctahedral $\mathrm{Pd}_{55}$ with a hydrogen atom. As shown in Figure 1, it is composed of eight triangular and six square faces that correspond to $\{111\}$ and $\{100\}$ facets. As larger size cluster gave almost same electronic properties with the $\mathrm{Pd}_{55}-\mathrm{H}$ model at least for surface-subsurface region, it is safe to 
use the results of the $\mathrm{Pd}_{55}-\mathrm{H}$ model for chemical discussion. With the weak cluster size dependency for the electronic states, we can also conclude that the cubooctahedral $\mathrm{Pd}_{55}$ can model surface environments of Pd nano-octahedrons with $\{111\}$ facet and $\mathrm{Pd}$ nano-cubes with $\{100\}$ facet in a proper manner. Using the $\mathrm{Pd}_{55}-\mathrm{H}$ model, we calculated the minimum energy paths for hydrogen absorption from (111) and (100) surfaces. First, $\mathrm{Pd}_{55}$ were optimized with cubooctahedral symmetry. Then, with fixing coordinates of the optimized $\mathrm{Pd}_{55}$, we constructed an $\mathrm{H}$-interacting $\mathrm{Pd}_{55}$ model. Changing the coordinate of the hydrogen atom with a Cartesian interval of $0.25 \AA$, total energies of $\mathrm{Pd}_{55}-\mathrm{H}$ were evaluated. In this study, we placed a hydrogen atom on 1800 symmetrically unique cubic grid points. Finally, H-interaction energy was obtained using the following equation

$$
\mathrm{E}_{\text {int }}=\mathrm{E}\left(\mathrm{Pd}_{55}-\mathrm{H}\right)-\left[\mathrm{E}\left(\mathrm{Pd}_{55}\right)+\mathrm{E}(\mathrm{H})\right]+1 / 2 \mathrm{D}_{0}\left(\mathrm{H}_{2}\right)
$$

where $E(X)$ is total energy of species $X$ and $D_{0}\left(H_{2}\right)$ is dissociation energy of a hydrogen molecule (4.48 eV [6]), which is subtracted to take into account dissociative absorption process (1) discussed in the previous paragraph. Using the total energies at the 1800 points, minimum energy paths for hydrogen absorption were obtained for $\{111\}$ and $\{100\}$ facets.

The second theoretical approach applied in this study is molecular dynamics (MD) simulation. For the MD simulations ReaxFF module implemented in ADF2014 program package [12] was applied. In order to compare hydrogen diffusion behavior in $\mathrm{Pd}$ nano-octahedron and $\mathrm{Pd}$ nano-cube, we applied a set of MD simulations with ReaxFF reactive force field, which can describe interaction among $\mathrm{Pd}$ and $\mathrm{H}$ atoms 
semi-quantitatively [13]. In the MD simulations, octahedral $\mathrm{Pd}_{891}$ and cubic $\mathrm{Pd}_{864}$ shown in Figure 2 were chosen as basic models for Pd nano-octahedron and Pd nano-cube particles, respectively. To calculate diffusion coefficients of hydrogen atoms in the Pd nano particle models, we placed hydrogen atoms randomly in the Pd nano particle models. As apparent difference in hydrogen absorbing rate has been observed at low hydrogen concentration condition, we set concentration of hydrogen to be $0.05,0.10$ and $0.15 \mathrm{H} / \mathrm{Pd}$ for each model. 10 different initial configurations were prepared for each hydrogen concentration. For each model mentioned above, first, we performed a conjugate gradient energy minimization to reduce simulation artifacts caused by high-energy contacts in the initial geometry. Then, a set of subsequent thermal equilibration run for $1 \mathrm{ps}$ was performed at $300 \mathrm{~K}$. Thirdly, a set of production MD run for 9 ps was performed for each model with the same temperature condition. For whole MD simulations, the NVT ensemble was applied and the time step was set to 0.25 fs. Berendsen thermostat [14] with a damping constant of 100 fs maintained temperature control throughout the simulation. Using the Einstein's equation, diffusion coefficient in each Pd nano particle model was calculated.

$$
\mathrm{D}=\frac{\sum_{\mathrm{i}}^{\mathrm{N}}\left\langle\left|\mathbf{r}_{\mathrm{i}}\left(\mathrm{t}+\mathrm{t}_{0}\right)-\mathbf{r}_{\mathrm{i}}\left(\mathrm{t}_{0}\right)\right|^{2}\right\rangle}{6 \mathrm{t}}
$$

where $\mathrm{D}, \mathrm{t}, \mathrm{t}_{0}, \mathrm{~N}$, and $\mathbf{r}_{\mathrm{i}}(\mathrm{t})$ are diffusion coefficient of hydrogen atoms, time, initial time for sampling of hydrogen diffusion, number of hydrogen atoms, and coordinates of i-th hydrogen atom, respectively. Similarly, we performed a set of production MD runs for each model with each hydrogen concentration at different temperature conditions $(400,500,600$, 
and $700 \mathrm{~K}$ ). The diffusion barrier of hydrogen atoms in each model was evaluated using the Arrhenius relationship

$$
\mathrm{D}=\mathrm{D}_{0} \exp \left(-\frac{\mathrm{E}_{\mathrm{a}}}{\mathrm{RT}}\right)
$$

where $\mathrm{D}_{0}, \mathrm{E}_{\mathrm{a}}$ and $\mathrm{R}$ are the Arrhenius pre-exponential factor, diffusion barrier, and the gas constant, respectively. The diffusion constant analyses explained above treat hydrogen atom diffusion in all regions inside Pd nano particles. Besides, using properly selected initial hydrogen configurations, we can also analyze differences in hydrogen diffusion kinetics between subsurface and inside (bulk) regions of Pd nano particles separately (See

Figure 3). Hereafter, we call the models without region decomposition "Whole" model. We also define the models for describing hydrogen diffusion within subsurface and bulk regions as "Sub" and "Bulk", respectively. Since almost all the hydrogen atoms move within the subsurface or bulk regions of Pd nano particle throughout simulation time, it is possible to understand differences in hydrogen diffusion kinetics between subsurface and bulk regions using the "Sub" and "Bulk" models.

\section{Results and Discussion}

\section{3-1. Hydrogen invasion from the surface to the subsurface of Pd nano particles}

A set of hydrogen absorption potential energy surface for cubooctahedral $\mathrm{Pd}_{55}$ model is depicted in Figure S1 in the supplemental material. Corresponding minimum energy paths for hydrogen absorption along [111] and [100] direction are given in Figure 4. 
In Figure 4, the horizontal axis means distance between each surface of the model Pd cluster and absorbed hydrogen atom. Negative value for horizontal axis corresponds to the situation that a hydrogen atom is absorbed in the model cluster. For [111] direction, an energy minimum corresponding to the hydrogen adsorption sites on the $\{111\}$ facet of the cluster model was found at $+0.80 \AA$. At the energy minimum, a hydrogen atom was adsorbed to a 3-fold hollow site on the $\{111\}$ surface of the model Pd cluster with hydrogen adsorption energy of $-0.96 \mathrm{eV}$. Similarly, for [100] direction, a local energy minimum assigned to hydrogen adsorption to 4 -fold hollow site on the $\{100\}$ facet was predicted at $1.17 \AA$. Hydrogen adsorption energy at the minimum was predicted to be $-0.88 \mathrm{eV}$.

As shown in Figure 4, we could also find a set of energy minima corresponding to hydrogen absorption. These minima could be assigned to tetrahedral $\left(\mathrm{T}_{\mathrm{d}}\right)$ or octahedral $\left(\mathrm{O}_{\mathrm{h}}\right)$ hydrogen absorption sites in the model cluster, which have been well known for hydrogen absorption in bulk Pd. Our minimum energy hydrogen invasion path analysis predicted that a hydrogen atom could reach an $\mathrm{O}_{\mathrm{h}}$ site at $-0.70 \AA$ directly when a hydrogen atom absorbed in the cluster from the $\{111\}$ surface. Although slight minima with interaction energy of $-0.49 \mathrm{eV}$ was found at $-0.10 \AA$ in the hydrogen absorption path along [111] direction, an energy barrier between the minima was small $(0.16 \mathrm{eV})$ and the interaction energy at the barrier is negative. The interaction energy at the $\mathrm{O}_{\mathrm{h}}$ site in the subsurface region along the direction was predicted to be $-0.52 \mathrm{eV}$.

On the other hand, when a hydrogen atom is absorbed in the cluster from $\{100\}$ surface, it was predicted that it is not possible for a hydrogen atom to reach an $\mathrm{O}_{\mathrm{h}}$ site of the Pd cluster in a direct manner. Along [100] direction, there is a $T_{d}$ hydrogen absorption site 
between the cluster surface and an $\mathrm{O}_{\mathrm{h}}$ site inside cluster. The $\mathrm{T}_{\mathrm{d}}$ and $\mathrm{O}_{\mathrm{h}}$ sites were found at -0.83 and $-1.58 \AA$ inside from the $\{100\}$ surface with the interaction energies of -0.11 and $-0.32 \mathrm{eV}$, respectively. As the hydrogen interaction energy between the $\mathrm{T}_{\mathrm{d}}$ and $\mathrm{O}_{\mathrm{h}}$ sites becomes almost zero, we could assign the $\mathrm{T}_{\mathrm{d}}$ site to a metastable hydrogen absorption site. For bulk Pd, it has been well known that $\mathrm{O}_{\mathrm{h}}$ site is more stable than $\mathrm{T}_{\mathrm{d}}$ sites. Since the crystal structure of the bulk Pd is well preserved in the Pd nano cluster, it is also natural for $\mathrm{Pd}_{55}$ that $\mathrm{O}_{\mathrm{h}}$ sites are more stable than $\mathrm{T}_{\mathrm{d}}$ sites. Since there is no thick potential barrier between the $T_{d}$ and the $O_{h}$ sites, it should be easy for a hydrogen atom to diffuse into inner $O_{h}$ site from outer $T_{d}$ site. Summarizing the above discussion, we can characterize the two hydrogen absorption paths as follows.

\section{Path along [111] direction:}

Adsorption of an $\mathrm{H}$ atom in 3-fold hollow site on surface

$\rightarrow \quad$ Direct invasion of an $\mathrm{H}$ atom into $\mathrm{O}_{\mathrm{h}}$ site

\section{Path along [100] direction:}

Adsorption of an $\mathrm{H}$ atom in 4-fold hollow site on surface

$\rightarrow \quad$ Invasion of an $\mathrm{H}$ atom into $\mathrm{T}_{\mathrm{d}}$ site

$\rightarrow \quad$ Rapid hydrogen diffusion of an $\mathrm{H}$ atom into $\mathrm{O}_{\mathrm{h}}$ site

It should be also noted that the energy barriers from surface to subsurface region were predicted to be 0.66 and $0.94 \mathrm{eV}$ for [111] and [100] paths, respectively (See Figure 4). 
These energy differences in the surface and subsurface regions show the fact that hydrogen adsorption/invasion paths along [111] direction should be more favorable than [100] in Pd nano particle, even though the predicted energy barriers did not take into account surface relaxation effects, which make both barriers lower.

\section{3-2 MD simulation for Hydrogen diffusion in Pd nanoparticle}

The hydrogen diffusion coefficients obtained by ReaxFF-MD simulations using models of $\mathrm{Pd}$ nano-octahedron $\left(\mathrm{Pd}_{891}\right)$ and $\mathrm{Pd}$ nano-cube $\left(\mathrm{Pd}_{864}\right)$ are shown in Figure 5. In Figure 5, hydrogen diffusion coefficients for "Whole", "Sub", and "Bulk" models are plotted for comparison.

First, we shall focus on differences in hydrogen diffusion coefficients in subsurface and bulk regions for both Pd nano-octahedron and Pd nano-cube. For Pd nano-octahedron, which is enclosed by $\{111\}$ facets, it was found that hydrogen diffusion in subsurface region is faster than those in bulk region at any hydrogen atom concentration. The hydrogen diffusion constants for "Sub" model of Pd nano-octahedron were predicted to be $2.54,2.90$ and $2.92 \times 10^{-8} \mathrm{~m}^{2} \mathrm{~s}^{-1}$ at the hydrogen concentration of $0.05,0.10$ and $0.15 \mathrm{H} / \mathrm{Pd}$, respectively. Corresponding values for "Bulk" model are 2.38, 1.91 and $1.94 \times 10^{-8} \mathrm{~m}^{2} \mathrm{~s}^{-1}$ at the same hydrogen concentration conditions. On the other hand, for Pd nano-cube, which is enclosed by $\{100\}$ facets, it was theoretically predicted that hydrogen diffusion coefficients within subsurface region are smaller than those in bulk region. The hydrogen diffusion constants for "Sub" model of Pd nano-cube are 1.32, 1.49 and $1.94 \times 10^{-8} \mathrm{~m}^{2} \mathrm{~s}^{-1}$, and those for "Bulk" model are 1.52, 1.70 and $1.82 \times 10^{-8} \mathrm{~m}^{2} \mathrm{~s}^{-1}$ at the hydrogen concentration of $0.05,0.10$ 
and $0.15 \mathrm{H} / \mathrm{Pd}$, respectively. These results show that the hydrogen diffusion behavior depends on cluster shape and hydrogen diffusion region. The reason why hydrogen diffusion constants show concentration dependency can be explained as follows. As mentioned in section 2, in the MD simulations of "Sub" and "Bulk" hydrogen diffusion models, only a few hydrogen atoms can jump between subsurface and bulk regions within the simulation time we applied. In other words, hydrogen atoms in "Sub" or "Bulk" models move within the subsurface or bulk regions of the Pd nano particles, respectively. As each interstitial site does not have same chemical environment even within a region due to atomic fluctuation by finite temperature effect, it is considered that the energy distribution of absorbed hydrogen should have a width. This means that the interstitial sites in each region of Pd nano particles are occupied by the order of energy stability. In general, diffusion coefficient is governed by the diffusion barrier between the interstitial sites. Thus, hydrogen atoms absorbed in relatively stable interstitial sites are less likely to diffuse into other interstitial sites than those in unstable interstitial site, i.e., diffusion coefficient of such stable hydrogen is small. At low hydrogen concentration, hydrogen atoms occupy stable site, and the diffusion constants are generally small. As seen Table 1, it was found that diffusion coefficients of hydrogen atoms are in direct proportion to hydrogen concentration except bulk region of Pd nano-octahedron model. A set of Arrhenius plots and diffusion barrier heights for Pd nano-octahedron and Pd nano-cube are shown in Figure 6 and Table 2, respectively. Our MD simulations showed that the barrier is $2.83 \mathrm{kcal} \mathrm{mol}^{-1}$ for hydrogen diffusion among $\mathrm{O}_{\mathrm{h}}$ sites in subsurface of $\mathrm{Pd}$ nano-octahedron. The barrier height is 0.72 $\mathrm{kcal} \mathrm{mol}^{-1}$ lower than that of Pd nano-cube. These results well explain the experimental 
results by Li et al., which showed that $\mathrm{Pd}$ nano-octahedron absorbs hydrogen atoms much faster than Pd nano-cube at low hydrogen concentration. In Table 2, it is also shown that chemical environments in the bulk regions for $\mathrm{Pd}$ nano-octahedron and $\mathrm{Pd}$ nano-cube are similar with each other. Using DFT calculations, Ozawa et al. showed that hydrogen absorption potentials in subsurface region of bulk Pd model (Pd slab model) do not show dependency on layer thickness [17]. Their results indicated that physicochemical environments for hydrogen absorption are almost constant inside subsurface region of $\mathrm{Pd}$ slab, which corresponds to "Bulk" region in our model. The small energy difference between $\mathrm{Pd}$ nano-octahedron and $\mathrm{Pd}$ nano-cube shows that the difference in hydrogen absorption potential is almost localized in subsurface region of $\mathrm{Pd}$ nano particles.

\section{Conclusion}

The difference in the hydrogen absorption kinetics between Pd nano-octahedrons with $\{111\}$ facet and Pd nano-cubes with $\{100\}$ facets was theoretically investigated using DFT and MD simulations. Both simulation results showed that the rate-determining steps

of hydrogen absorption for the Pd nano particles are hydrogen atom invasion process from surface to subsurface. It was found that the hydrogen absorption barrier heights between surface and subsurface are reflected to the shape dependency of hydrogen absorption kinetics.

\section{Acknowledgments}


This work was supported by CREST project, Japan Science and Technology agency (JST). A part of calculations reported here were performed using computing resources in the Research Center for Computational Science, Okazaki, Japan. One of the author (A.M.) has been supported by Japan society for the promotion of science (JSPS) scholarship for young researcher (JSPS KAKENHI Grant Number 13J10731). We are grateful for these supports. 


\section{References}

[1] M. Yamauchi, R. Ikeda, H. Kitagawa, M. Takata, J. Phys. Chem. C 112 (2008) 3294.

[2] M. Yamauchi, H. Kobayashi, H. Kitagawa, ChemPhysChem 10 (2009) 2566.

[3] H. Kobayashi, M. Yamauchi, H. Kitagawa, Y. Kubota, K. Kato, M. Takata, J. Am. Chem. Soc. 130 (2008) 1828.

[4] B. Lim, M. Jiang, J. Tao, P. H. C. Camargo, Y. Zhu, Y. Xia, Adv. Funct. Mater. 19 (2009) 189.

[5] G. Li, H. Kobayashi, S. Dekura, R. Ikeda, Y. Kubota, K. Kato, M. Takata, T. Yamamoto, S. Matsumura, H. Kitagawa, J. Am. Chem. Soc. 136 (2014) 10222.

[6] K. Christmann, Surf. Sci. Rep. 9 (1988) 1.

[7] TURBOMOLE V6.4 2012, a development of University of Karlsruhe and Forschungszentrum Karlsruhe GmbH, 1989-2007,TURBOMOLE GmbH, since 2007; available from http://www.turbomole.com.

[8] J. P. Perdew, K. Burke, M. Ernzerhof, Phys. Rev. Lett. 77 (1996) 3865.

[9] J. P. Perdew, K. Burke, M. Ernzerhof, Phys. Rev. Lett. 78 (1997) 1396.

[10] K. Eichkorn, O. Treutler, H. Öhm, M. Häser, R. Ahlrichs, Chem. Phys. Lett. 240 (1995) 283

[11] W. H. Freeman, Thermal Physics (2nd ed.). San Francisco ISBN 978-0-7167-1088-2. (1980) 357.

[12] ADF2014, SCM, Theoretical Chemistry, Vrije Universiteit, Amsterdam, The Netherlands, http://www.scm.com.

[13] T. P. Senftle, M. J. Janik, A. C. T. van Duin, J. Phys. Chem. C 118 (2014) 4967. 
[14] H. J. C. Berendsen, J. P. M. Postma, W. F. van Gunsteren, A. DiNola, J. R. Haak, J. Chem. Phys. 81 (1984) 3684.

[15] S. Hara, A. Caravella, M. Ishitsuka, H. Suda, M. Mukaida, K. Haraya, E. Shimano, T. Tsuji, J. Membr. Sci. 421-422 (2012) 355.

[16] K. Nishimura, J. Soc. Mater. Sci., Japan 63 (2014) 163.

[17] N. Ozawa, T. A. Roman, H. Nakanishi, H. Kasai, N. B. Arboleda, W. A. Diño, J. Appl. Phys. 101 (2007) 123530. 
Table 1. Hydrogen diffusion coefficients at the low hydrogen concentration conditions $\left(0.05-0.15 \mathrm{H} / \mathrm{Pd}\right.$; in units of $\left.\times 10^{-8} \mathrm{~m}^{2} \mathrm{~s}^{-1}\right)$.

\begin{tabular}{|c|c|c|c|c|c|c|}
\hline \multirow{2}{*}{$\mathrm{H} / \mathrm{Pd}$} & \multicolumn{2}{|c|}{ Whole model } & \multicolumn{2}{|c|}{ Sub model } & \multicolumn{2}{|c|}{ Bulk model } \\
\hline & $\{111\}$ & $\{100\}$ & $\{111\}$ & $\{100\}$ & $\{111\}$ & $\{100\}$ \\
\hline 0.05 & 3.10 & 1.59 & 2.54 & 1.32 & 2.38 & 1.52 \\
\hline 0.10 & 2.28 & 1.56 & 2.90 & 1.49 & 1.91 & 1.70 \\
\hline 0.15 & 2.25 & 1.58 & 2.92 & 1.94 & 1.94 & 1.82 \\
\hline
\end{tabular}


Table 2. Diffusion barriers obtained from the Arrhenius plots (in units of kcal mol ${ }^{-1}$ ).

\begin{tabular}{|c|c|c|c|c|}
\hline Region & $\begin{array}{c}\text { Pd nano-octahedron } \\
(\{111\} \text { facet })\end{array}$ & \multicolumn{1}{c}{$\begin{array}{c}\text { Pd nano-cube } \\
(\{100\} \text { facet })\end{array}$} & \multicolumn{1}{c}{$\begin{array}{c}\text { Exp. } \\
{[15]}\end{array}$} & $\begin{array}{c}\text { MD } \\
\text { (Bulk Pd) [16] }\end{array}$ \\
\hline Subsurface & 2.83 & 3.55 & \multirow{2}{*}{5.04} & 3.69 \\
\hline Inside (Bulk) & 3.06 & 3.01 & & \\
\hline
\end{tabular}




\section{Figure Captions}

Figure 1 Model cluster used for DFT calculations in this study.

Figure 2 Pd nano-octahedron and Pd nano-cube models applied to our ReaxFF MD simulation.

Figure 3 Definition of subsurface and inside (bulk) region (the case of $\mathrm{Pd}$ nano-octahedron).

Figure 4 Minimum energy paths for hydrogen absorption from (a) $\{111\}$ and (b) $\{100\}$ surfaces of $\mathrm{Pd}_{55}$.

Figure 5 Hydrogen concentration dependency of hydrogen diffusion coefficients in (a) "Whole", (b) "Sub", and (c) "Bulk" models for Pd nano-octahedron and Pd nano-cube.

Figure 6 The Arrhenius plots for hydrogen diffusion constants for (a) subsurface of Pd nano-octahedron, (b) inside (bulk) of Pd nano-octahedron, (c) subsurface of Pd nano-cube, and (d) inside (bulk) of Pd nano-cube. 


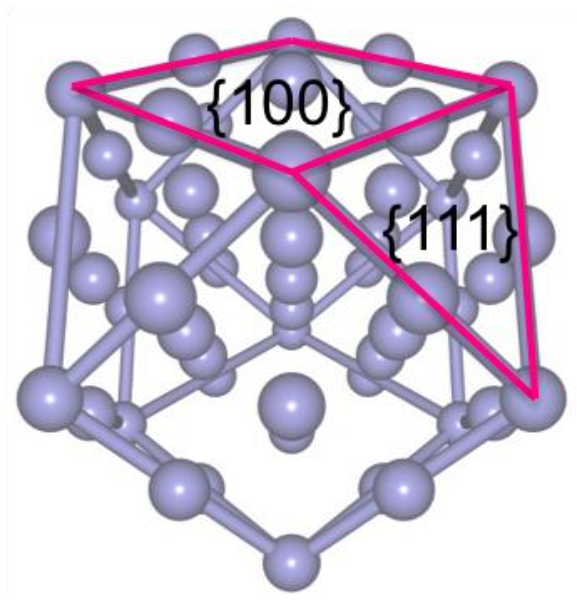

Figure 1 Matsuda et al. 

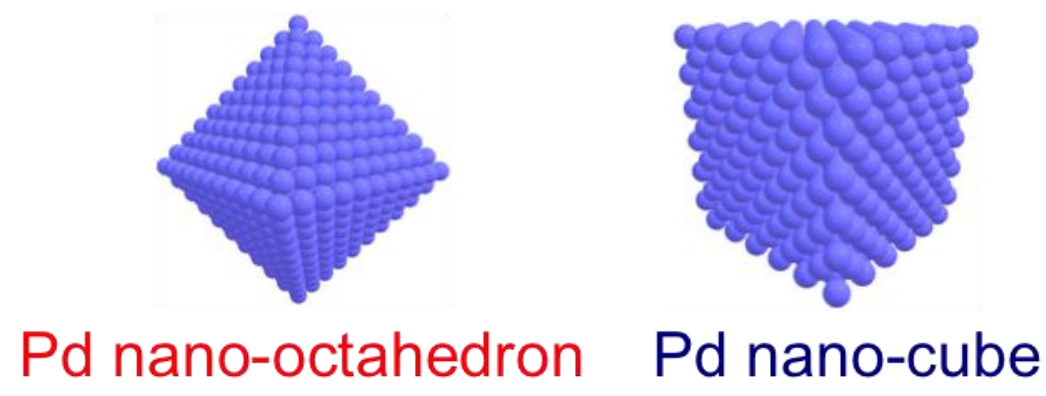

Figure 2 Matsuda et al. 


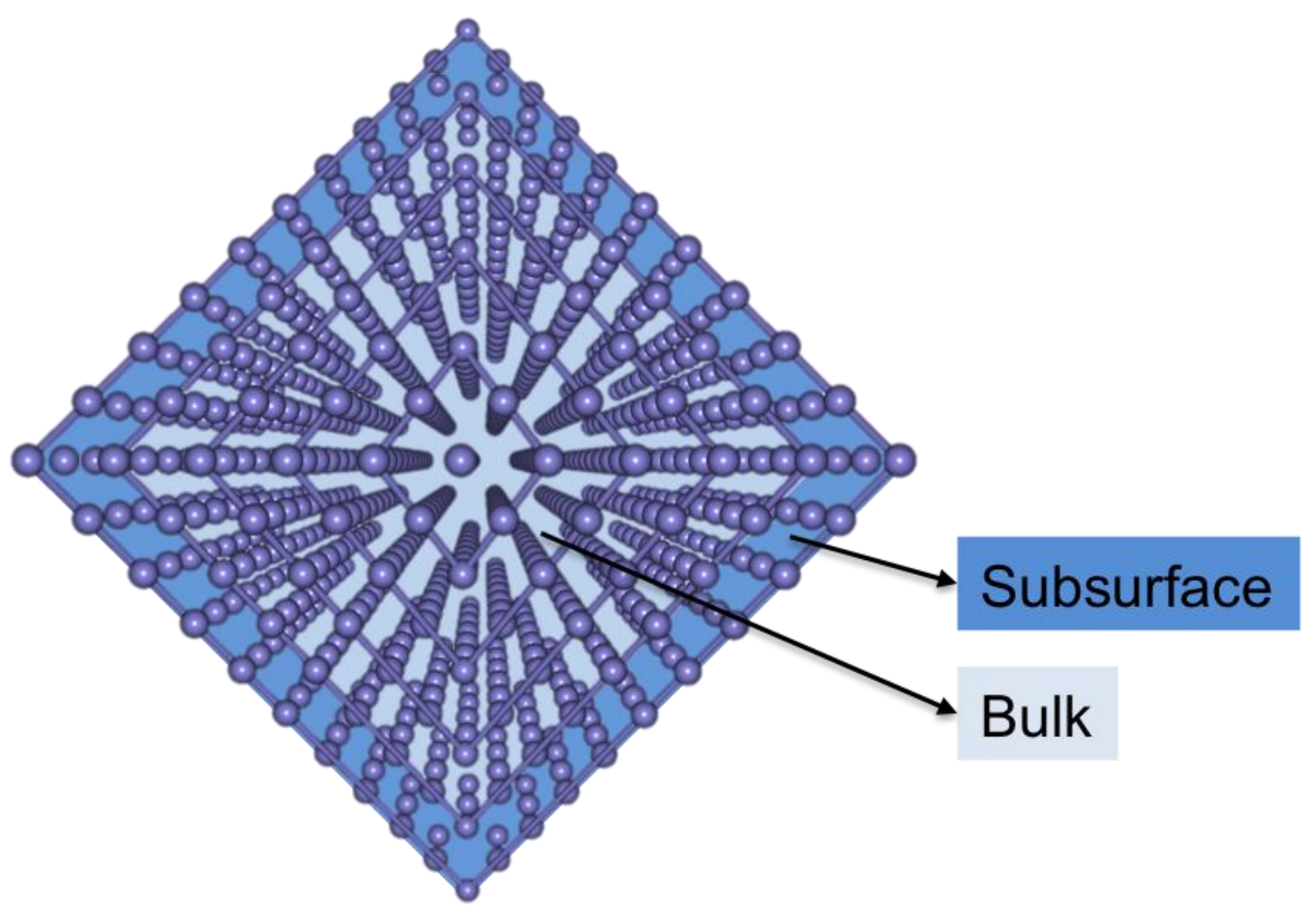

Figure 3 Matsuda et al. 

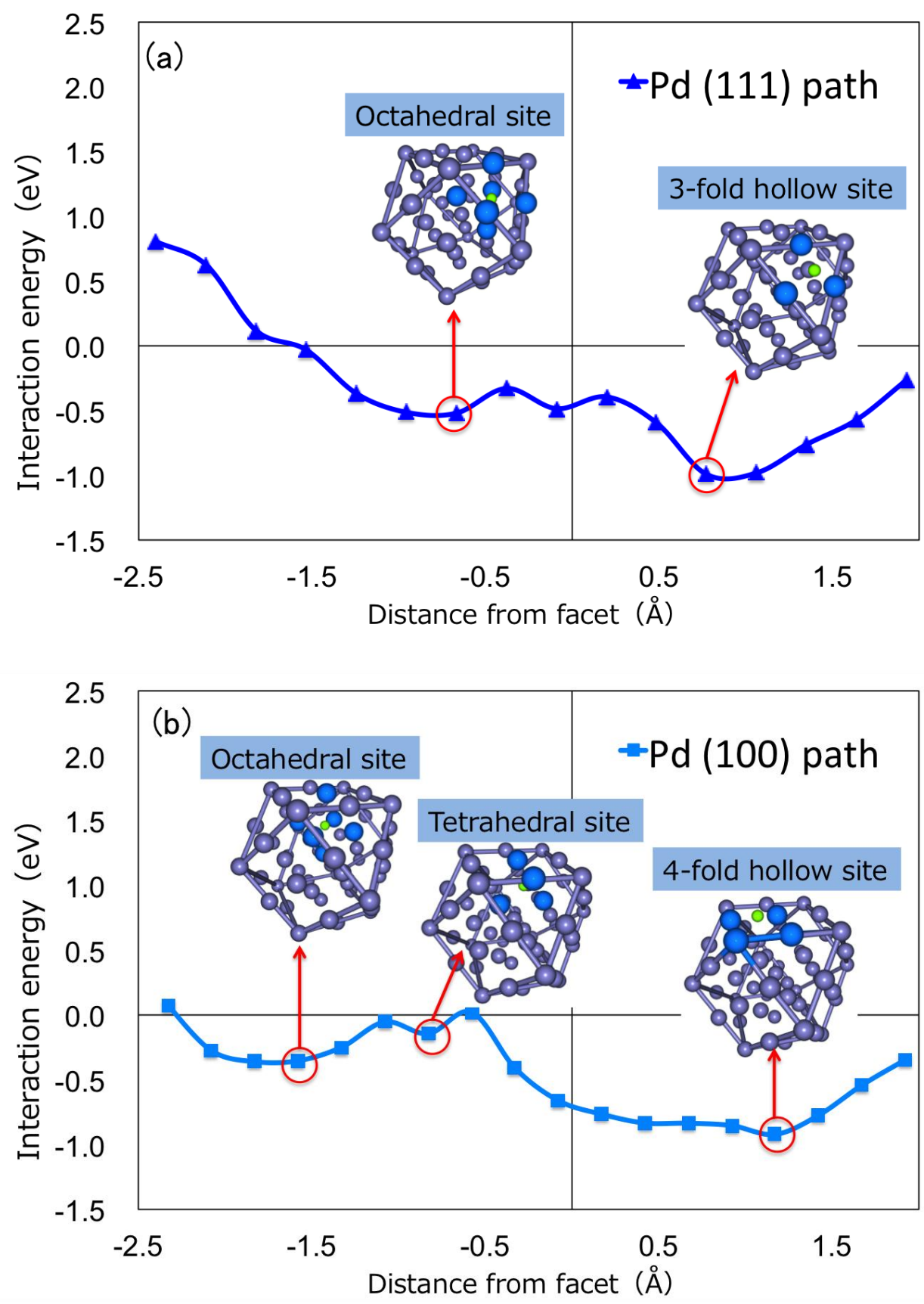

Figure 4 Matsuda et al. 

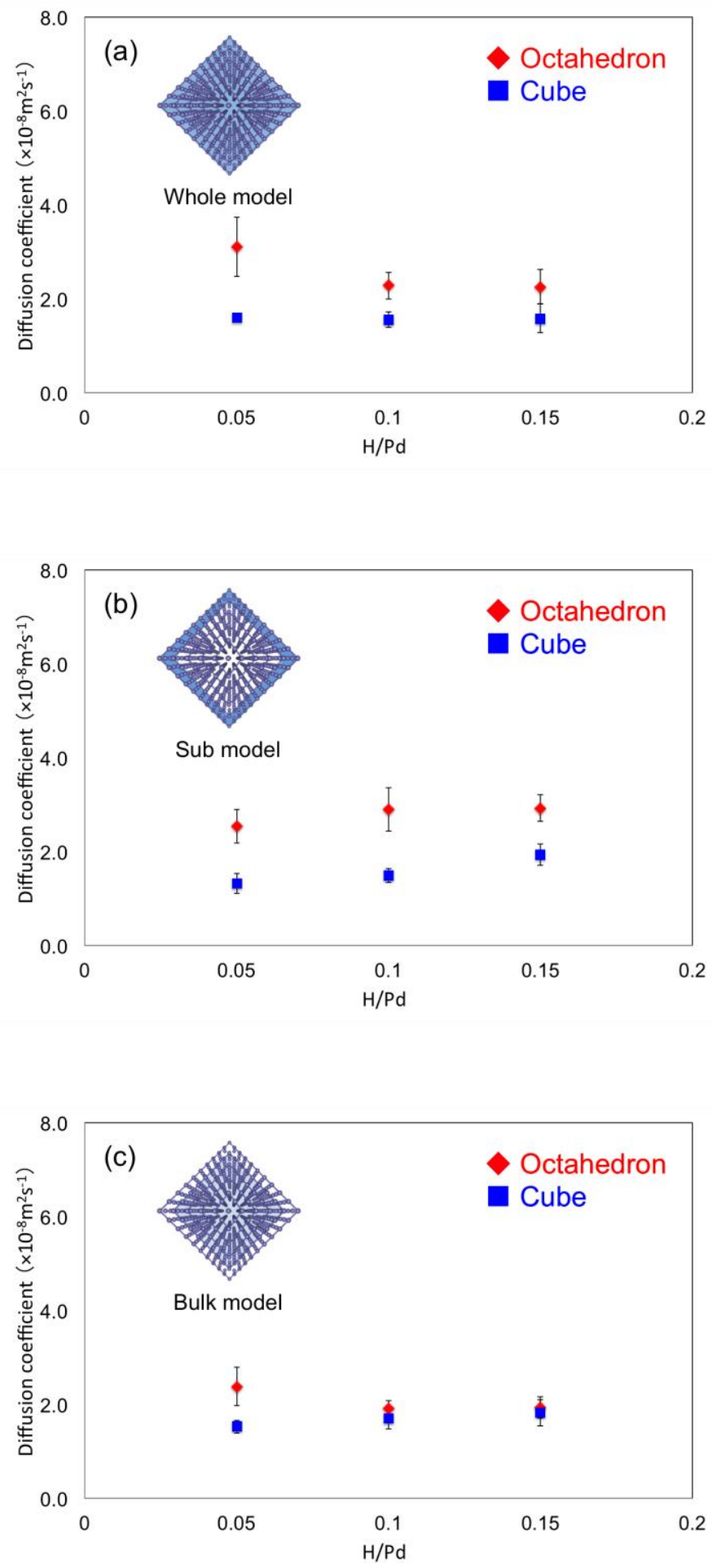

Figure 5 Matsuda et al. 

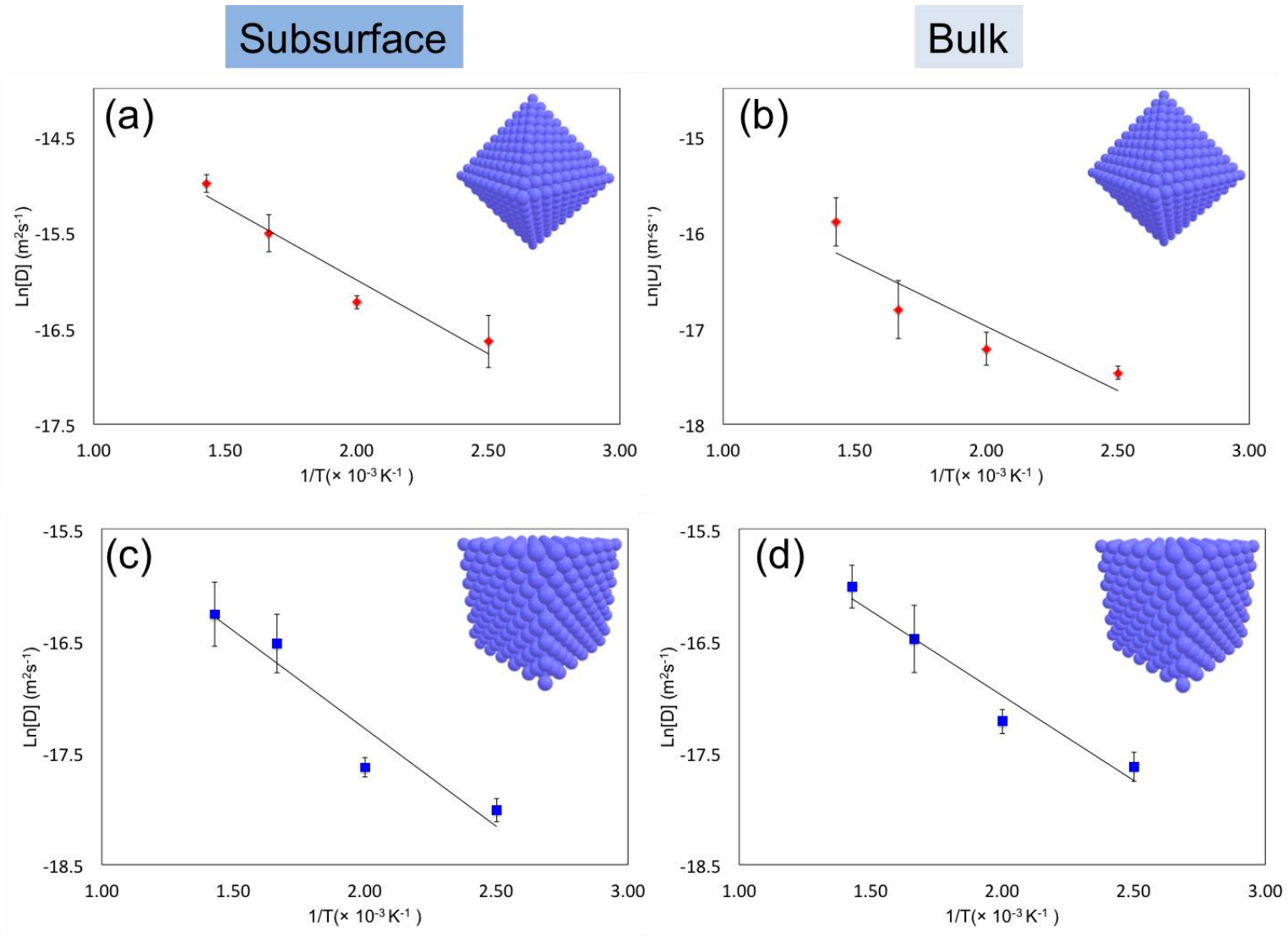

Figure 6 Matsuda et al. 


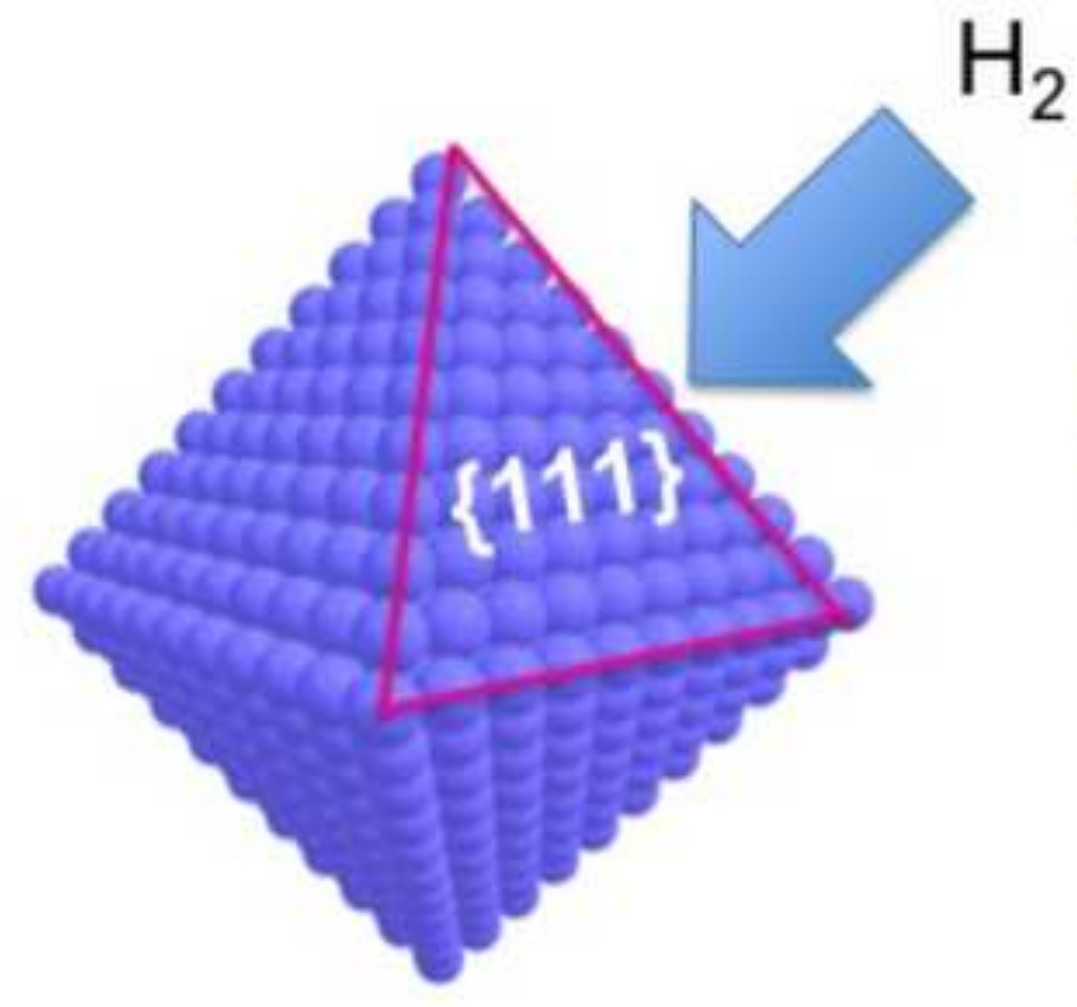

Pd nano-octahedron

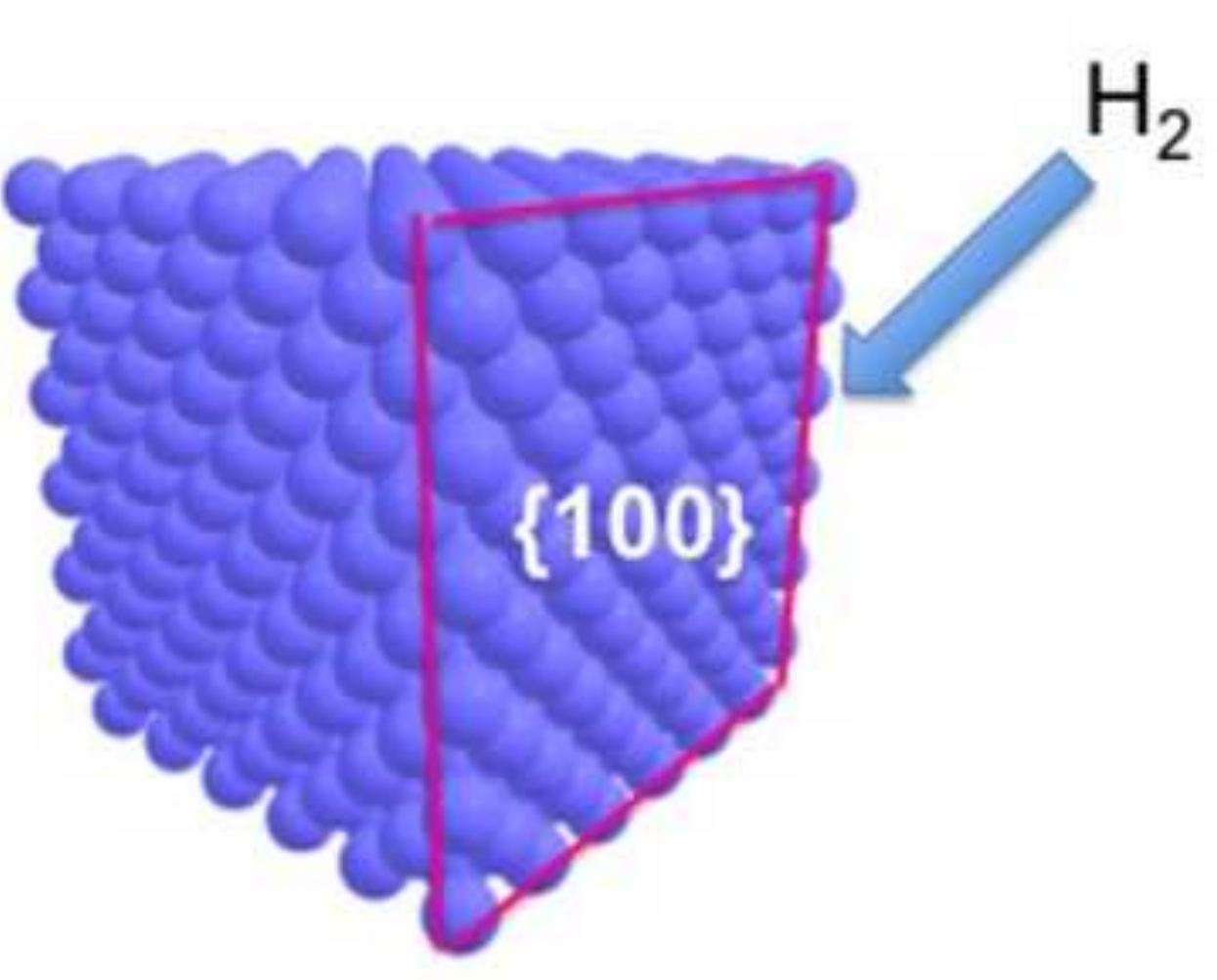

Pd nano-cube

\section{Larger hydrogen absorption rate for} Pd nano-octahedron ( $\{111\}$ facet) can be explained by difference in surface electronic states. 